\title{
ON TOTAL MASSES OF BALAYAGED MEASURES
}

\author{
MASAYUKI ITÔ
}

\section{Introduction}

Beurling and Deny [1], [2] introduced the notion of Dirichlet spaces. They [2] showed the existence of balayaged measures and equilibrium measures in the theory of Dirichlet spaces. In this paper, we shall show that the following equivalence is valid for a Dirichlet space on a locally compact Hausdorff space $X$.

(1) For a pure potential $u_{\mu}$ such that $S_{\mu}$, the support of $\mu$, is compact and for a. compact neighborhood $\omega$ of $S_{\mu}$, let $\mu^{\prime}$ be the balayaged measure of $\mu$ to $\mathscr{C} \omega$. Then

$$
\int d \mu=\int d \mu^{\prime}
$$

(2) For an increasing net $\left(\omega_{\alpha}\right)_{\alpha \varepsilon I}$ of relatively compact open sets satisfying $\omega_{\alpha} \nearrow X$, let $\nu_{\alpha}$ be the equilibrium measure of $\omega_{\alpha}$. Then the net $\left(\nu_{\alpha}\right)_{\alpha \in I}$ converges vaguely to 0 .

Furthermore we shall examine anologous equivalences for a special Dirichlet space on a locally compact abelian group $X$.

\section{Preliminaries on Dirichlet spaces}

According to Beurling and Deny [2], we define a normal contraction of the complex plane $\mathfrak{c}$.

Definition 1. A transformation $T$ of $\mathbb{C}$ into itself is called a normal contraction if it satisfies the following conditions:

$$
T(0)=0 \text { and }\left|T z_{1}-T z_{2}\right| \leq\left|z_{1}-z_{2}\right|
$$

for any couple of $z_{1}$ and $z_{2}$ in $\mathfrak{c}$.

Let $X$ be a locally compact Hausdorff space and let $C_{k}=C_{k}(X)$ be the space of complex valued continuous functions with compact support provided with the topology of uniform convergence.

Definition 2.1) Let $\xi$ be a positive Radon measure in $X$ which is every-

Received May 6, 1966.

1) Cf. [2], p. 209. 
where dense (i.e., $\xi(\omega)>0$ for each non-empty open set $\omega$ in $X$ ). A Hilbert space $D=D(X, \xi)$ is called a $\xi$-Dirichlet space (simply, a Dirichlet space) if each element in $D$ is a complex valued function $u(x)$ which is locally summable for $\xi$ and the following three conditions are satisfied.

a) For each compact subset $K$ in $X$, there exists a positive constant $A(K)$ such that, for any $u$ in $D$

$$
\int_{K}|u(x)| d \xi(x) \leq A(K)\|u\| .
$$

b) $C_{k} \cap D$ is dense both in $C_{k}$ and in $D$.

c) For any normal contraction $T$ and any $u$ in $D$,

$$
T u \varepsilon D \text { and }\|T u\| \leq\|u\| \text {. }
$$

More precisely, two functions which are equal to $p . p$. in $X^{2)}$ represent the same element in $D$. The norm in $D$ is denoted by $\|\cdot\|$, the associated scalar product by $(\cdot, \cdot)$.

Definition 3.3) An element $u$ in $D$ is called a potential if there exists a Radon measure $\mu$ such that

$$
(u, v)=\int \bar{v} d \mu
$$

for any $v$ in $C_{k} \cap D$. Such an element $u$ is denoted by $u_{\mu}$. Especially if $\mu$ is positive, $u_{\mu}$ is called a pure potential.

It is evident that the subspace of linear combinations of pure potentials is dense in $D$.

Definition 4.4) We say that a property holds p.p.p. on a subset $E$ in $X$ if the property holds $\mu-p . p$. for any pure potential $u_{\mu}$ such that $S_{\mu} \subset E .^{5}$ )

It is evident that a property holds $p . p$. on a subset $E$ in $X$ if the property holds p.p.p. on $E$, because for any complex valued bounded measurable function $f$ with compact support, there exists the potential $u_{f}$ generated by $f$.

In order to prove our main theorem, we need the following lemmas. Let $D$ be a Dirichlet space on $X$. For each element $u$ in $D$, the refinement of $u$ is

2) A property is said to hold p.p. in a subset $E$ in $X$ if the property holds in $E$ except a set which is locally of $\xi$-measure 0 .

3) Cf. [2], p. 209.

4) Cf. [7].

5) $S_{\mu}$ is the support of $\mu$. 
denoted by $\left.u^{*} .{ }^{6}\right)$

Lemma 1. For elements $u$ and $v$ in $D$, suppose that $u(x) \geq v(x) p . p$. in an open set $G$. Then $u^{*}(x) \geq v^{*}(x)$ p.p.p. in $G$.

Proof. For any pure potential $u_{\mu}$ such that $S_{\mu} \subset G$, it is sufficient to prove that

$$
\left(u, u_{\mu}\right) \geq\left(v, u_{\mu}\right)
$$

because

$$
\left(u, u_{\mu}\right)=\int u^{*} d \mu \text { and }\left(v, u_{\mu}\right)=\int v^{*} d \mu .
$$

Similarly as in the proof of Lemma 3 in [7], there exist positive bounded measurable functions $f_{n}$ with compact support such that $f_{n}(x)=0$ p.p. in $\mathscr{C} G$ and the sequence $\left(u_{f_{n}}\right)$ converges weakly $u_{\mu}$ in $D$. By our assumption,

$$
\left(u, u_{f_{n}}\right)=\int u(x) f_{n}(x) d \xi \geq \int v(x) f_{n}(x) d \xi=\left(v, u_{f_{n}}\right)
$$

Hence

$$
\left(u, u_{\mu}\right)=\lim _{n \rightarrow \infty}\left(u, u_{f_{n}}\right) \geq \lim _{n \rightarrow \infty}\left(v, u_{f_{n}}\right)=\left(v, u_{\mu}\right) .
$$

This completes the proof.

By Lemma 1, we obtain the following domination theorem.

Lemma 2. For pure potentials $u_{\mu_{1}}$ and $u_{\mu_{2}}$ in $D$, suppose that

$$
u_{\mu_{1}}(x) \geq u_{\mu_{2}}(x)
$$

p.p. in some open neighborhood $\omega$ of $S_{\mu_{2}}$. Then

$$
u_{\mu_{1}} \geq u_{\mu_{2}}
$$

Proof. By Lemma 1,

$$
u_{\mu_{1}}^{*}(x) \geq u_{\mu_{2}}^{*}(x)
$$

p.p.p. in $\omega . \quad$ It is known that there exists a pure potential $u_{\nu}$ such that ${ }^{7)}$

$$
u_{\nu}=\inf \left(u_{\mu_{1}}, u_{\mu_{2}}\right) \text {. }
$$

6) Cf. [2], p. 210.

7) Cf. [4], Lemma 2, p. 5. 
By above, it holds that

$$
\begin{aligned}
& u_{\nu}^{*}(x)=\mu_{\mu_{2}}^{*}(x) \quad \text { p.p.p. in } \omega, \\
& u_{\nu}^{*}(x) \leq u_{\mu_{2}}^{*}(x) \quad \text { p.p.p. in } X .
\end{aligned}
$$

Then we have

$$
\begin{aligned}
& \left\|u_{\mu_{2}}-u_{\nu}\right\|^{2}=\left\|u_{\mu_{2}}\right\|^{2}-2\left(u_{\mu_{2}}, u_{\nu}\right)+\left\|u_{\nu}\right\|^{2} \\
& =\int u_{\mu_{2}}^{*} d \mu_{2}-2 \int u_{\nu}^{*} d \mu_{2}+\int u_{\nu}^{*} d \nu \\
& =\int u_{\nu}^{*} d \nu-\int u_{\nu}^{*} d \mu_{2} \\
& =\int\left(u_{\nu}^{*}-u_{\mu_{2}}^{*}\right) d \nu \leq 0
\end{aligned}
$$

Hence

$$
u_{\mu_{2}}=u_{\nu} \text {, i.e., } u_{\mu_{1}} \geq u_{\mu_{2}} .
$$

This completes the proof.

By the above lemma, we obtain the following unicity theorem.

Corollary. Let $u_{\mu_{1}}$ and $u_{\mu_{2}}$ be two potentials in $D$. If

$$
u_{\mu_{1}}(x)=u_{\mu_{2}}(x) \text { p.p. }
$$

in some neighborhood of $S_{\mu_{1}} \cup S_{\mu_{2}}$, then $\mu_{1}=\mu_{2}$.

This is evident by Lemma 2.

Lemma 3. For elements $u$ and $v$ in $D$, the following equalities hold.

$$
\begin{array}{ll}
(\alpha u+\beta v) *(x)=\alpha u^{*}(x)+\beta v^{*}(x) & \text { p.p.p. in } X, \\
(\inf (u, v))^{*}(x)=\inf \left(u^{*}(x), v^{*}(x)\right) & \text { p.p.p. in } X .
\end{array}
$$

The proof is evident by Lemma 1 and the fact that $\left(u^{*}\right) *(x)=u^{*}(x) p \cdot p \cdot p$. in $X$ for any $u$ in $D$.

Lemma 4. For any pure potential $u_{\mu}$ in $D$ with $\int d \mu<+\infty$ and any positive number $M$, there exists a pure potential $u_{\mu_{M}}$ such that

$$
u_{\mu_{M}}(x)=\inf \left(u_{\mu}(x), M\right) \text { and } \int d \mu_{M} \leq \int d \mu .
$$

Proof. The existence of a pure potential $u_{\mu_{M}}$ is followed from a result of 
Deny. ${ }^{8)}$ For a relatively compact open set $\omega$, let $u_{\nu}$ be the equilibrium potential of $\omega .{ }^{9)}$ Then

$$
\begin{aligned}
& \int_{\omega} d \mu_{M} \leq \int u_{\nu}^{*} d \mu_{M}=\left(u_{\nu}, u \mu_{M}\right)=\int u_{\mu_{M}}^{*} d \nu \\
& =\int \inf \left(u_{\mu}^{*}(x), M\right) d \nu \leq \int u_{\mu}^{*} d \nu \\
& =\int u_{\nu}^{*} d \mu \leq \int d \mu .
\end{aligned}
$$

$\omega$ being arbitrary, we obtain

$$
\int d \mu_{M} \leq \int d \mu .
$$

This completes the proof.

Now we define the spectrum of an element in $D$. Given an element $u$ in $D$, there exists the greatest open set $\omega$ having the following property: $(u, v)=0$ for any $v$ in $C_{k} \cap D$ with support in $\omega$.

Definition 5.9) The complementary set of such an open set $\omega$ is called the spectrum of $u$, denoted by $s(u)$.

Evidently for any potential $u_{\mu}$ in $D, s\left(u_{\mu}\right)=S_{\mu}$.

We put, for an open set $\omega$,

$$
\begin{aligned}
& D_{\omega}^{(1)}=\{\overline{u \varepsilon D ; s(u) \subset \omega}\}, \\
& D_{\omega}^{(2)}=\left\{f_{\varepsilon} C_{k} \cap D ; S_{f} \subset \omega\right\},
\end{aligned}
$$

and for a closed set $F$ in $X$,

$$
\begin{aligned}
& D_{F}^{(1)}=\{u \varepsilon D ; s(u) \subset F\}, \\
& D_{F}^{(3)}=\left\{u \varepsilon D ; u^{*}(x)=0 \quad \text { p.p.p. on } F\right\} .
\end{aligned}
$$

Lemma 5. Let $u_{\mu}$ be a pure potential in $D$ and let $F$ be a closed set in $X$. Then there exists a pure potential $u_{\mu^{\prime}}$ in $D$ such that

$$
\begin{aligned}
& \mu^{\prime} \text { is supported by } F \text { and } \int d \mu^{\prime} \leq \int d \mu, \\
& u_{\mu}^{*}(x)=u_{\mu^{\prime}}^{*}(x) \quad \text { p.p.p. on } F, \\
& u_{\mu}(x) \geq u_{\mu^{\prime}}(x) \quad \text { p.p. in } X
\end{aligned}
$$

8) Cf. [4], p. 6.

9) Cf. [2], p. 215. 


$$
u_{\mu^{\prime}} \text { is equal to the projection of } u_{\mu} \text { to } D_{F}^{(1)} \text {. }
$$

Proof. We put

$$
E_{u_{\mu^{\prime}}} F=\left\{u \varepsilon D ; u^{*}(x) \geq u_{\mu}^{*}(x) \quad \text { p.p.p. on } F\right\} .
$$

Then $E_{u_{\mu}, F}$ is a non-empty closed convex set in $D$. Let $u^{\prime}$ be the unique element which minimizes the norm in $E_{u_{\mu}, F}$. Similarly as the proof of Beurling and Deny's Balayage Theorem, ${ }^{10}$ ) we can prove that $u^{\prime}$ is a pure potential in $D$ and the conditions (1)-(3) are satisfied. Furthermore similarly as the proof of Lemma 3 in [7], we can prove that the condition (4) is satisfied.

We remark that for a pure potential $u_{\mu}$ in $D$, the element which satisfied the conditions (1)-(3) is uniquely determined in $D$ by Lemma 2. We call such a pure potential $u_{\mu^{\prime}}$ the balayaged potential of $u_{\mu}$ to $F$ and the positive measure $\mu^{\prime}$ the balayaged measure of $\mu$ to $F$.

Lemma 6. For an open set $\omega$ in $X, D_{\omega}^{(2)}$ is a Dirichlet space on $\omega$ with the norm induced from the norm in $D$. Let $u_{\mu}^{\prime}$ be a pure potential in $D_{\omega}^{(2)}$ such that $S_{\mu}$ is compact in $\omega$. Then there exists a potential $u_{\mu}$ in $D$ such that

$$
u_{\mu}^{\prime}(x)=u_{\mu}(x)-u_{\mu^{\prime}}(x),
$$

where $u_{\mu^{\prime}}$ is the balayaged potential of $u_{\mu}$ to $\mathscr{C} \omega$.

Proof. It is evident that $D_{\omega}^{(2)}$ become a Dirichlet space on $\omega$ by the norm induced from the norm in $D$. We may assume that

$$
D_{\omega}^{(2)}=\left\{u-u_{1} ; u \varepsilon D_{\omega}^{(1)}\right\},
$$

where $u_{1}$ is the projection of $u$ to $D_{\mathscr{C} \omega}^{(1)}$. Hence there exists an element $v$ in $D_{\omega}^{(1)}$ such that

$$
u_{\mu}^{\prime}=v-v_{1}
$$

Obviously

$$
s\left(v-v_{1}\right) \subset s_{\mu} \cup \mathscr{C} \omega .
$$

$S_{\mu}$ being compact in $\omega, s(v)=S_{\mu}$ and for any $\varphi$ in $C_{k} \cap D_{\omega}^{(2)}$,

$$
(v, \varphi)=\int \bar{\varphi}(x) d \mu
$$

that is, for any $\varphi$ in $C_{k} \cap D$,

$$
(v, \varphi)=\int \bar{\varphi}(x) d \mu .
$$

10) Cf. [2], p. 210 and [7]. 
Therefore $v=u_{\mu}$ and by Lemma 5, $v_{1}=u_{\mu^{\prime}}$. This completes the proof.

\section{Main theorems}

By the above lemmas, we obtain the following main theorems.

Theorem I. Let $D$ be a Dirichlet space on $X$. Then the following two conditions are equivalent.

(I. 1) For a pure potential $u_{\mu}$ in $D$ such that $S_{\mu}$ is compact in $X$ and a compact neighborhood $\omega$ of $S_{\mu}$, let $\mu^{\prime}$ be the balayaged measure of $\mu$ to $\mathscr{C} \omega .{ }^{11)}$ Then

$$
\int d \mu=\int d \mu^{\prime}
$$

(I. 2) For an increasing net $\left(\omega_{\alpha}\right)$ of relatively compact open sets with $\omega_{\alpha} \nearrow X$, the net of the equilibrium measures $\nu_{\alpha}$ of $\omega_{\alpha}{ }^{12)}$ converges vaguely to 0 .

Proof. First we prove the implication (I. 1) $\Rightarrow\left(\right.$ I. 2). Since the net $\left(u_{\nu_{\alpha}}^{*}\right)$ is increasing and converges to 1 p.p.p. in $X$,

$$
\lim _{\alpha \in I} \int u_{\nu_{\alpha}}^{*} d \mu=\lim _{\alpha \in I} \int u_{\nu_{\alpha}}^{*} d \mu^{\prime}
$$

for any $u_{\mu}$ in $D$ such that $S_{\mu}$ is compact and any compact neighborhood $\omega . S_{\mu}$ being compact,

$$
0=\lim _{\alpha \in I} \int u_{\nu_{\alpha}}^{*} d\left(\mu-\mu^{\prime}\right)=\lim _{\alpha \in I} \int\left(u_{\mu}^{*}-\mu_{\mu^{\prime}}^{*}\right) d \nu_{\alpha} .
$$

We take a fixed function $\varphi$ in $C_{k}$. Next we take a relatively compact open sets $\omega$ and $\omega_{0}$ such that

$$
S_{\varphi} \subset \omega_{0} \subset \bar{\omega}_{0} \subset \omega .
$$

Let $u_{\nu}^{\prime}$ be the equilibrium potential of $\omega_{0}$ in the Dirichlet space $D_{\omega}^{(2)}$. By Lemma 6 , there exists a pure potential $u_{\nu}$ in $D$ such that

$$
u_{\nu}^{\prime}=u_{\nu}-u_{\nu^{\prime}},
$$

where $u_{\nu}$ is the balayaged potential of $u_{\nu}$ to $C \omega$. Furtheremore we take a relatively compact open set $\omega^{\prime}$ such that $\bar{\omega} \subset \omega^{\prime}$. Let $\nu^{\prime \prime}$ be the balayaged measure of $\nu$ to $\mathscr{C} \bar{\omega}^{\prime}$. Then

$$
u_{\nu^{\prime}}(x) \geq u_{\nu^{\prime \prime}}(x)
$$

11) Cf. [2], p. 210 and [7].

12) Cf. [2], p. 210 and [7]. 
p.p. in $\mathscr{C} \omega . \quad$ By Lemma 2,

$$
u_{\nu^{\prime}}(x) \geq u_{\nu^{\prime \prime}}(x)
$$

$p . p$. in $X$. That is,

$$
u_{\nu}^{*}(x)-u_{\nu^{\prime}}^{*}(x) \leq u_{\nu}^{*}(x)-u_{\nu^{\prime \prime}}^{*}(x)
$$

p.p.p. in $X$. Hence there exists a positive number $M$ such that

$$
\begin{aligned}
& \varlimsup_{\alpha \in I} \int|\varphi| d \nu_{\alpha} \leq \varlimsup_{\alpha \varepsilon I} M \int\left(u_{\nu}^{*}-u_{\nu^{\prime}}^{*}\right) d \nu_{\alpha} \\
& \leq \varlimsup_{\alpha \in I} M \int\left(u_{\nu}^{*}-u_{\nu^{\prime \prime}}^{*}\right) d v_{\alpha}=\lim _{\alpha \in I} M \int\left(u_{\nu}^{*}-u_{\nu^{\prime \prime}}^{*}\right) d \nu_{\alpha}=0 .
\end{aligned}
$$

Therefore the net $\left(\nu_{\alpha}\right)_{\alpha \varepsilon I}$ converges vaguely to 0 .

Next we prove the implication (I. 2) $\Rightarrow$ (I. 1). Let $u_{\mu}$ and $u_{\mu^{\prime}}$ be the elements in our theorem. By Lemma 4, for each positive number $M$, there exists positive measures $\mu_{M}$ and $\mu_{M}^{\prime}$ such that

$$
u_{\mu_{M}}=\inf \left(u_{\mu}, M\right) \text { and } u_{\mu_{M}^{\prime}}=\inf \left(u_{\mu^{\prime}}, M\right) \text {. }
$$

Since we have

$$
\int d \mu_{M} \leq \int d \mu \text { and } \int d \mu_{M}^{\prime} \leq \int d \mu^{\prime},
$$

we may assume that there exist bounded linear functionals $T$ and $T^{\prime}$ on $C$, where $C$ is the Banach space of bounded continuous functions in $X$ with norm

$$
\|f\|_{C}=\sup _{x \in X}|f(x)| .
$$

Then

$$
\int f d \mu_{M} \longrightarrow T(f) \text { and } \int f d \mu_{M}^{\prime} \longrightarrow T^{\prime}(f)
$$

as $M \rightarrow \infty$ for any $f$ in $C$. On the other hand

$$
\left\|u_{\mu_{M}}\right\|^{2}=\int u_{\mu_{M}}^{*} d \mu_{M} \leq \int u_{\mu}^{*} d \mu_{M}=\int u_{\mu_{M}}^{*} d \mu \leq \int u_{\mu}^{*} d \mu=\left\|u_{\mu}\right\|^{2}
$$

Similarly we have

$$
\left\|u_{\mu_{M}^{\prime}}\right\|^{2} \leq\left\|u_{\mu^{\prime}}\right\|^{2} .
$$

For any bounded measurable function $f$ with compact support, we have the following convergences 


$$
\begin{aligned}
& \lim _{M \rightarrow \infty} \int u_{\mu_{M}}(x) f(x) d \xi(x)=\int u_{\mu}(x) f(x) d \xi(x), \\
& \lim _{M \rightarrow \infty} \int u_{\mu_{M}^{\prime}}(x) f(x) d \xi(x)=\int u_{\mu^{\prime}}(x) f(x) d \xi(x) .
\end{aligned}
$$

Hence $\left(u_{\mu_{M}}\right)$ and $\left(u_{\mu_{M}^{\prime}}\right)$ converges weakly to $u_{\mu}$ and $u_{\mu^{\prime}}$, respectively, because the totality of potentials generated by such functions $f$ is dense in $D$. Now for any $\varphi$ in $C_{k} \cap D$,

$$
T(\varphi)=\int \varphi d \mu \text { and } T^{\prime}(\varphi)=\int \varphi d \mu^{\prime} .
$$

By the denseness of $C_{k} \cap D$ in $C_{k}$,

$$
T(f) \geq \int f d \mu \quad \text { and } \quad T^{\prime}(f) \geq \int f d \mu^{\prime}
$$

for any $f$ in $C$. On the othr hand by lemma 4 ,

$$
T(1) \leq \int d \mu \quad \text { and } \quad T^{\prime}(1) \leq \int d \mu^{\prime} .
$$

That is,

$$
T(1)=\int d \mu \quad \text { and } \quad T^{\prime}(1)=\int d \mu^{\prime}
$$

By the above equality, it is sufficient to prove that for any $M>0$,

$$
\int d \mu_{M}=\int d \mu_{M}^{\prime}
$$

Since we have

$$
\begin{aligned}
& 0 \leq u_{\mu_{M}}^{*}-u_{\mu_{M}^{\prime}} \leq M \quad \text { p.p.p. in } X, \\
& u_{\mu_{M}}^{*}-u_{\mu_{M}^{\prime}}^{*}=0 \quad \text { p.p.p. in } \mathscr{C} \omega,
\end{aligned}
$$

there exists a function $\varphi$ in $C_{k}$ such that

$$
u_{\mu_{M}}^{*}(x)-u_{\mu_{M}^{\prime}}^{*}(x) \leq \varphi(x)
$$

$p . p . p$. in $X$. Hence by our assumption,

$$
\varlimsup_{\alpha \in I} \int^{2}\left(u_{\mu_{M}}^{*}-u_{\mu_{M}^{\prime}}^{*}\right) d \nu_{\alpha} \leq \lim _{\alpha \in I} \int \varphi d \nu_{\alpha}=0
$$

Therefore

$$
\lim _{\alpha \in I} \int\left(u_{\mu_{M}}^{*}-u_{\mu_{M}^{\prime}}^{*}\right) d \nu_{\alpha}=\lim _{\alpha \in I} \int u_{\nu_{\alpha}}^{*} d\left(\mu_{M}-\mu_{M}^{\prime}\right)=0
$$


That is,

$$
\int d \mu_{M}=\int d \mu_{M}^{\prime}
$$

This completes the proof.

Remark 1. In the above theorem, a sufficient condition for the condition (I. 1) to be satisfied is the following $\left(\mathbf{I} \cdot 2^{\prime}\right)$.

(I. $\left.2^{\prime}\right) \quad$ There exists an increasing net $\left(\omega_{\alpha}\right)_{\alpha \in I}$ of relatively compact open sets such that $\omega_{\alpha} \nearrow X$ and the net of the equilibrium measures $\nu_{\alpha}$ of $\omega_{\alpha}$ converges vaguely to 0.

Remark 2. Let $X$ be a bounded domain in the $n$-dimensional Euclidean space $R^{n}(n \geq 2)$ with sufficiently smooth boundary and let $0<\alpha<2$. In the Dirichlet space $D_{\alpha}^{0}$ on $X$ introduced by Elliott [6], the condition (I. 1) in Theorem I is not satisfied, because for any sequence $\left(\omega_{m}\right)$ of relatively compact open sets tending to $X$, the sequence $\left(\nu_{m}\right)$ of the equilibrium measures of $\omega_{m}$ converges vaguely to $m(x)$, where

$$
m(x)=J_{\alpha} \int_{\mathscr{C} X}|x-y|^{-\alpha-n} d y
$$

and $J_{\alpha}$ is a positive constant.

By Beurling and Deny's Representation Theorem ${ }^{13)}$ and our Theorem I, we obtain the following

Theorem II. Let $D$ be a Dirichlet space on $X$. Then the following two conditions are equivalent.

(II. 1) For a pure potential $u_{\mu}$ in $D$ such that $S_{\mu}$ is compact and a compact neighborhood $\omega$ of $S_{\mu^{\prime}}$, let $\mu^{\prime}$ be the balayaged measure of $\mu$ to $\mathscr{C} \omega$. Then

$$
\int d \mu=\int d \mu^{\prime}
$$

(II. 2) There exist a positive Hermitian form $N(f, g)$ on $C_{k} \cap D$ with a local character ${ }^{14)}$ and a positiveve symmetric measure $\sigma$ in $X \times X-\delta(\delta$ is the diagonal set of $X \times X)$ such that

$$
(f, g)=N(f, g)+\iint(f(x)-f(y))(\bar{g}(x)-\bar{g}(y)) d \sigma(x, y) .
$$

By Beurling and Deny's Representation Theorem and the remark with respect to it in [7], it is evident that the conditions (I. 1) and (II. 2) are equivalent.

13) Cf. [2], pp. 211 and [7].

14) This means that $N(f, g)=0$ if $g$ is constant in some neighborhood of $S_{f}$. 


\section{Special Dirichlet spaces}

According to Beurling and Deny [2], we define a negative definite function in a locally compact abelian group $X$ and a special Dirichlet space on $X$.

Definition 6.15) A complex valued continuous function $\lambda(x)$ defined in $X$ is said to be negative definite if the following Hermitian form

$$
\sum_{i, j=1}^{n}\left(\lambda\left(x_{i}\right)+\overline{\lambda\left(x_{j}\right)}-\lambda\left(x_{i}-x_{j}\right)\right) \rho_{i} \rho_{j}
$$

is positive for each set of $n$ points $x_{1}, x_{2}, \ldots \ldots, x_{n}$ in $X$ and each $n$ complex number $\rho_{1}, \rho_{2}, \ldots \ldots, \rho_{n}(n=1,2, \ldots \ldots)$.

Definition 7.16) A Dirichlet space $D=D(X, \xi)$ is said to be special if $X$ is a locally compact abelian group and $\xi$ is the Haar measure on $X$, the following condition being satisfied.

d) If $U_{x} u$ is the function obtained from $u$ in $D$ by the translation $x \varepsilon X$ (i.e., $\left.U_{x} u(y)=u(y-x)\right)$, then

$$
U_{x} u \varepsilon D \text { and }\left\|U_{x} u\right\|=\|u\| \text {. }
$$

Buerling and Deny [2] showed the following important result.

To a special Dirichlet space $D$ on $X$ corresponds a real valued negative definite function $\lambda(\hat{x})$ on the dual group $\hat{X}$ of $X$ such that $\lambda^{-1}$ is locally summable and the following equality holds:

$$
\|\left. u\right|^{2}=\int \lambda(\hat{x})|\hat{u}(\hat{x})|^{2} d \hat{x}
$$

for any $u$ in $C_{k} \cap D$, where $\hat{\mathrm{u}}$ is the Fourier transform of $u$.

Conversely, such a negative definite function $\lambda(\hat{x})$ on $\hat{X}$ defines, by means of (1), a special Dirichlet space on $X$.

Furthermore for a special Dirichlet space $D$, there exists a positive measure $\kappa$ having $\lambda^{-1}$ as the Fourier transform. We call this measure $\kappa$ the kernel of $D$. We [7] proved the following proposition.

Proposition. Let $D$ be a special Dirichlet space on $X$ and let $\kappa$ be the kernal of $D$. For a point $x$ in $X$ and a compact neighborhood $\omega$ of $x$, there exists a positive measure $\varepsilon_{x}^{\prime}$ such that

15) Cf. [2], p. 214 and [4], p. 8.

16) Cf. [2], p. 215 and [4], p. 9. 


$$
\begin{aligned}
& \varepsilon_{x}^{\prime} \text { supported by } \overline{\mathscr{C} \omega} \text { and } \int d \varepsilon_{x}^{\prime} \leq 1, \\
& \kappa * \varepsilon_{x}=\kappa * \varepsilon_{x}^{\prime} \text { as a measure in } \mathscr{C} \omega, \\
& \kappa * \varepsilon_{x} \geq \kappa * \varepsilon_{x}^{\prime} \text { in } X .
\end{aligned}
$$

This meaure $\varepsilon_{x}^{\prime}$ is called the balayaged measure of the unit measure $\varepsilon_{x}$ at $x$ to $\mathscr{C} \omega$.

To prove the second main theorem, we need the following lemmas.

Lemma 7. Let $D$ be a special Dirichlet space on $X$. For each increaesing net $\left(\omega_{\alpha}\right)_{\alpha \varepsilon I}$ of relatively compact open sets with $\omega_{\alpha} \nearrow X$, the net $\left(\nu_{\alpha}\right)_{\alpha \varepsilon I}$ converges vaguely to $\lambda(\hat{o})$ if $\lambda(\hat{o}) \neq 0$, where $\nu_{\alpha}$ is the equilibrium measure of $\omega_{\alpha}$.

Cf. [7], Lamma 12.

Lemma 8. Let $D_{1}$ and $D_{2}$ be special Dirichlet spaces on $X$ and $\lambda_{1}(\hat{x})$ and $\lambda_{2}(\hat{x})$ be the negative definite functions of $D_{1}$ and $D_{2}$, respectively. If $\lambda_{1}(\hat{x}) \geq \lambda_{2}(\hat{x})$, then $D_{1} \subset D_{2}$.

Proof. For any $u$ in $C_{k} \cap D_{1}$,

$$
\|u\|_{2}^{2}=\int|\hat{u}(\hat{x})|^{2} \lambda_{2}(\hat{x}) d \hat{x} \leq \int|\hat{u}(\hat{x})|^{2} \lambda_{1}(\hat{x}) d \hat{x}=\|u\|_{1}^{2},
$$

where $\|\cdot\|_{i}$ is the norm in $D_{i}$. Then $u$ is in $D_{2}$ and $\|u\|_{2} \leq\|u\|_{1}$.

Therefore $D_{1} \subset D_{2}$, because $C_{k} \cap D_{1}$ is dense in $D_{1}$. This completes the proof.

By Lemma 7 and Lemma 8, we obtain the following

Lemma 9. Let $D$ be a special Dirichlet space on $X$ and let $\lambda(\hat{x})$ be the negative definite function of $D$. For each increasing net $\left(\omega_{\alpha}\right)_{\alpha \varepsilon I}$ of relatively compact open sets with $\omega_{\alpha} \nearrow X$, the net $\left(\nu_{\alpha}\right)_{\alpha \in I}$ converges vaguely to $\lambda(\hat{o})$, where $\nu_{\alpha}$ is the equilibrium measure of $\omega_{\alpha}$.

Proof. For a fixed positive number $c$, we put

$$
\lambda^{\prime}(\hat{x})=\lambda(\hat{x})+c .
$$

Then $\lambda^{\prime}$ is negative definite and $\lambda^{\prime-1}$ is finite continuous in $X$. Let $D^{\prime}$ be the special Dirichlet space on $X$ such that $\lambda^{\prime}(\hat{x})$ is the negative definite function of $D^{\prime}$. For our net $\left(\omega_{\alpha}\right)_{\alpha \varepsilon I}$, we take another net $\left(\omega_{\alpha \varepsilon I}^{\prime}\right)_{\alpha \varepsilon}$ of relatively compact open sets such that

$$
\overline{\omega_{\alpha}} \subset \omega_{\alpha}^{\prime}
$$

for any $\alpha$. Let $u_{\mu_{\alpha}}^{\prime}$ be the condensor potential with respect to $\omega_{\alpha}$ and $\overline{\mathscr{C} \omega_{\alpha}^{\prime}}$ in 
$D^{\prime}$. We take a fixed function $\varphi$ in $C_{k}$ such that

$$
\varphi(x) \geq 0 \text { and } \int \varphi(x) d x=1 .
$$

Then $u_{\mu_{\alpha} * \varphi}$ is in $C_{k} \cap D^{\prime}$. Put

$$
u_{\alpha}(x)=u_{\mu_{\alpha}} * \varphi(x)
$$

Then $u_{\alpha}(x)$ tends to 1 . For any $u$ in $C_{k} \cap D^{\prime}$, there exists a number $\alpha_{0}$ such that $u_{\alpha}(x)=1$ in some neighborhood of $S_{u}$ for each $\alpha \geq \alpha_{0}$. By Beurling and Deny's Representation Theorem and Lemma 7,

$$
\left(u, u_{\alpha}\right)^{\prime}=(\lambda(\hat{o})+c) \int u(x) d x+2 \iint u(x)\left(1-u_{\alpha}(y)\right) d \sigma(x, y),
$$

where $(\cdot, \cdot)^{\prime}$ is the scalar product in $D^{\prime}$ and $\sigma$ is a positive symmetric measure in $X \times X-\delta(\delta$ is the diagonal set of $X \times X)$. Hence we obtain that

$$
\begin{aligned}
& \lim _{\alpha \in I}\left(u, u_{\alpha}\right)^{\prime}=\lim _{\alpha \in I} \int u(x) \mu_{\alpha} * \varphi(x) d x \\
& =(\lambda(\hat{o})+c) \int u(x) d x .
\end{aligned}
$$

On the other hand by Beurling and Deny's Representation theorem and Lemma 8 , The net $\left(\nu_{\alpha}\right)$ converges vaguely to some positive measure $\nu$ and we obtain the following equality,

$$
\lim _{\alpha \varepsilon I}\left(u, u_{\alpha}\right)=\int u(x) d \nu(x)
$$

Since we have the equality

$$
\begin{aligned}
& \left(u, u_{\alpha}\right)^{\prime}-\left(u, u_{\alpha}\right)=c \int u(x) \overline{u_{\alpha}}(x) d x=c \int u(x) u_{\alpha}(x) d x, \\
& \lim _{\alpha \in I}\left(u, u_{\alpha}\right)=\int u(x) d \nu(x)=\lambda(\hat{o}) \int u(x) d x .
\end{aligned}
$$

By the denseness of $C_{k} \cap D^{\prime}$ in $C_{k}$, we have the equality $\nu=\lambda(\hat{o})$ as a measure in $X$. This completes the proof.

By Theorem I and the above lemma, we obtain the following theorem.

Theorem III. Let $D$ be a special Dirichlet space on $X$. Then the following three condition are equivalent.

(1) There exist a point $x$ in $X$ and a compact neighborhood $\omega$ of $x$ such that 


$$
\int d \varepsilon_{x}^{\prime}=1
$$

where $\varepsilon_{x}^{\prime}$ is the balayaged measure of $\varepsilon_{x}$ to $\mathscr{C} \omega$.

$$
\lambda(\hat{o})=0 .
$$

(3) For any point $x$ in $X$ and any compact neighborhood $\omega$ of $x$, the total mass of the balayaged measure of $\varepsilon_{x}$ to $\mathscr{C} \omega$ is equal to 1 .

Proof. First we shall prove the implication (1) $\Rightarrow(2)$. Assume that $\lambda(\hat{o}) \neq 0$. Then $\lambda^{-1}$ is finite continuous in $X$, because

$$
\lambda(\hat{x}) \geq \lambda(\hat{o})
$$

for all $\hat{x}$ in $\hat{X}$. By Bochner's theorem, the total mass of the kernel $\kappa$ of $D$ is finite. By the unicity theorem with respect to special Dirichlet spaces (Cf. [7]),

$$
\int d \kappa>\int d\left(\kappa * \varepsilon_{x}^{\prime}\right)=\int d \kappa \cdot \int d \varepsilon_{x}^{\prime}
$$

for each $x$ and each compact neighborhood $\omega$ of $x$. That is, the total mass of $\varepsilon_{x}^{\prime}$ is less than 1. This contradicts our assumption.

The implication (2) $\Rightarrow(3)$ is evidently followed from Theorem I and Lemma 9.

The implication $(3) \Rightarrow(1)$ is evident.

This completes the proof.

Moreover we obtain the following

Theorem IV. Let $D$ be a special Dirichlet space on $X$ and let $\lambda(\hat{x})$ be the negative definite function of $D$. Assume that $\lambda(\hat{o}) \neq 0$. Then for any increasing net $\left(\omega_{\alpha}\right)_{\alpha \in I}$ of compact neighborhoods of $x$ in $X$ with $\omega_{\alpha} \nearrow X$, we obtain the following convergence

$$
\lim _{\alpha \varepsilon I} \int d \varepsilon_{\alpha}^{\prime}=0
$$

where $\varepsilon_{\alpha}^{\prime}$ is the balayaged measrure of $\varepsilon_{x}$ to $\mathscr{C} \omega_{\alpha}$.

Proof. By our assumption, $\lambda(\hat{x})>0$ for any $\hat{x}$ in $X$. Hence the total mass of the kernel $\kappa$ of $D$ is finite. Since

$$
\int d \varepsilon_{\alpha}^{\prime}<1 \text { and } S_{\varepsilon_{\alpha}^{\prime}} \subset \overline{\mathscr{C} \omega_{\alpha}}
$$

we obtain the convergence 


$$
\lim _{\alpha \in I} \int f(x) d \varepsilon_{\alpha}^{\prime}=0
$$

for any finite continuous function $f$ vanishing at infinity. On the other hand

$$
u_{\mu_{\alpha}^{\prime}}(x) \geq u_{\mu_{\beta}^{\prime}}(x)
$$

if $\alpha \leq \beta$ for any pure potential $u_{\mu}$, where $u_{\mu_{\alpha}^{\prime}}$ and $u_{\mu_{\beta}^{\prime}}$ are the balayaged potentials of $u_{\mu}$ to $\mathscr{C} \omega_{\alpha}$ and $\mathscr{C} \omega_{\beta}$, respectively. Hence

$$
\kappa * \varepsilon_{\alpha}^{\prime} \geq \kappa * \varepsilon_{\beta}^{\prime}
$$

if $\alpha \leq \beta$. Since the total mass of $\kappa$ is finite, there exists a positive measure $\eta$ such that

$$
\lim _{\alpha \varepsilon I} \int f(x) d\left(\kappa * \varepsilon_{\alpha}^{\prime}\right)=\int f(x) d \eta
$$

for any bounded continouus function $f$ in $X$. For each $\varphi$ in $C_{k}, \kappa * \varphi(x)$ is a finite continuous function vanishing at infinity, and hence

$$
\lim _{\alpha \in I} \int \varphi(x) d\left(\kappa * \varepsilon_{\alpha}^{\prime}\right)=\lim _{\alpha \in I} \int \kappa * \varphi(x) d \varepsilon_{\alpha}^{\prime}=0 .
$$

Therefore $\eta=0$. Now since the total mass of $\kappa$ is not zero, there exists a bounded measurable function $f$ in $X$ such that

$$
\kappa^{*} f(x) \geq 1
$$

in $X$. Then

$$
\begin{aligned}
& \varlimsup_{\alpha \varepsilon I} \int d \varepsilon_{\alpha}^{\prime} \leq \varlimsup_{\alpha \varepsilon I} \int \kappa * f(x) d \varepsilon_{\alpha}^{\prime} \\
& =\varlimsup_{\alpha \in I} \int f(x) d\left(\kappa * \varepsilon_{\alpha}^{\prime}\right)=\lim _{\alpha \in I} \int f(x) d\left(\kappa * \varepsilon_{\alpha}^{\prime}\right)=0 .
\end{aligned}
$$

This completes the proof.

Remark. Let $D$ be a Dirichlet space on a locally compact Hausdorff space $X . \quad$ It is an open question if the same result with Theorem IV exists when $\nu_{\alpha}$, the equilibrium measure of $\omega_{\alpha}$, tends vaguely to a non-zero measure for an increasing net $\left(\omega_{\alpha}\right)$ of relatively compact open sets with $\omega_{\alpha} \nearrow X$. 


\section{REFERENCES}

[1] A. Beurling and J. Deny: Espaces de Dirichlet I, Le case élémentaire, Acta Math., 99 (1958), 203-214.

[2] A. Beurling and J. Deny: Dirlichlet spaces, Proc. Nat. Acad. U.S.A., 45(1959) 208-215.

[3] S. Bochner: Harmonic analysis and the theory of probablity, Berkeley, 1955.

[5] J. Deny: Sur les espaces de Dirichlet, Sémin. Théorie du potentiel, Paris, 1957.

[4] J. Deny: Principe complet du maximum et Contractions, Ann. Inst. Fourier, 15 (1965), 259-272.

[6] J. Elliott: Dirichlet spaces associated with intregro-differential operators Part I, Illinois Math. J., (1965), 87-98.

[7] M. Itô: Characterizations of supports of balayaged measures, Nagoya Math. J., 28 (1966), 203-230.

Mathematical Institute Nagoya University 